\title{
Multi-agent system based adaptive protection for dispersed generation integrated distribution systems
}

\author{
Chengxi Liu ${ }^{a^{*}}$, Zakir Hussain Rather ${ }^{\mathrm{a}, \mathrm{b}}$, Zhe Chen ${ }^{\mathrm{a}}$, Claus Leth Bak ${ }^{\mathrm{a}}$ \\ ${ }^{a}$ Department of Energy Technology, Aalborg University, 9220 Aalborg Denmark \\ ${ }^{b}$ Innovation Center, kk-electronic, a/s, 9220 Aalborg Denmark
}

\begin{abstract}
The increasing penetration of dispersed generation (DG) brings challenges to conventional protection approaches of distribution system, mainly due to bi-directional power flow and variable fault current contribution from different generation technology-based DG units. Moreover, the trend of allowing islanded operation of distribution systems necessitates the adoption of adaptive protection methods for distribution systems. In order to improve the reliability and selectivity of protection for such kind of distribution systems, a coordinative adaptive protection based on multiagent system (MAS) is proposed. The adaptive protection intelligently adopts suitable settings for the variation of fault current from diversified DG units. Furthermore, the structure of mobile MAS with additional flexibility is capable of adapting the changes of system topology in a short period, e.g. radial/meshed, grid-connected/islanded. The protection method is tested on a simplified distribution system model of Denmark in DIgSILENT/PowerFactory.
\end{abstract}

Keywords: Adaptive protection, dispersed generation, distribution protection, multi-agent system

\section{Introduction}

Due to scarcity of fossil fuel reserves and the energy policy of $\mathrm{CO}_{2}$ emission reduction, various types of renewable energy (RE) are being integrated into power systems at tremendous pace. Because of the decentralized characteristics of these natural resources, significant part of these RE-based generating units is connected to distribution systems. In Denmark, currently more than $40 \%$ of today's installed capacity is from DG units, which are mostly connected to grid at the distribution level (60/20/10/0.4 kV). Large scale RE-based and other DG units would play significant roles as power resources in the modernized power systems, which make the protection of power systems more complicated than that of traditional systems.

At distribution voltage level, one of the major challenges is the variability and uncertainty of short circuit current from these flexible DG resources. The operating conditions of renewable generation are highly unpredictable. Moreover, low voltage ride through (LVRT) capability mandates DG units' connectivity to the network only for a short period. Therefore, the protection settings of distribution system are of crucial importance, because it should be able to not only isolate the faulted part of the system in a short time to prevent large scale tripping of DG units, but also immediately adapt to the new scenarios to achieve high reliability and selectivity [1].

The other challenge is the change of network topology, such as disconnecting some DG units and meshing the distribution feeders. In future, the increasing penetration of DG is expected to support all loads in the distribution system and hence be possible for islanded operation. Compared with grid connected mode, the fault current seen by protection relays in islanded mode is relatively much less.

Adaptive protection is defined as "an online activity that modifies the preferred protection settings in response to changes in system condition or requirements in a timely manner by means of external generated signals or control actions" [2]. Many researchers have proposed both communication-based adaptive protection methods [3]-[8] and communication-less adaptive protection methods [9]-[14] for DG

* Manuscript received July 17, 2013; revised September 11, 2013.

Corresponding author Tel.: +45 2252 8660; Fax: +45 9815 1411. E-mail address: cli@et.aau.dk. 
integrated distribution systems. In this paper, multi-agent system (MAS) is proposed for adaptive protection schemes to deal with these variable scenarios after analyzing the impact of DG units on protection coordination. The mobile structure of MAS with more flexibility is capable of adapting the variation of renewable generation and the changes of system topologies in a short time, so as to correctly protect the system from multiple instantaneous faults.

\section{Test Distribution System}

Fig. 1 shows the single-line model of the test distribution system, which is a part of distribution system owned by Danish Distribution System Operator (DSO), Himmerland Elforsyning (HEF) in Aalborg, Denmark. The test system consists of 6 loads (numbered from Load1 to Load6) and 7 buses (numbered from Bus1 to Bus7). Relays are represented by symbols "Rij", where numbers $i$ and $j$ represent the beginning bus and the ending bus of its protection zone, respectively. Five Gas Turbine Generators (GTGs) and one variable speed wind turbine generator (WTG) are connected to the system. The WTG is a detailed model of $2 \mathrm{MW}$ doubly-fed induction generator (DFIG) based wind turbine with power electronic converters and other control models, e.g. aerodynamic model, mechanical model, etc.. The GTGs are 3.3 MVA with governor and excitation control. The penetration level of DG units is sufficient to support all local loads across the distribution network in islanded operation mode. Meanwhile, the distribution system is capable of forming a ring type topology by Line 67 equipped with circuit breakers (CBs) to support the important load, (i.e. Load 5). R67 and R76 control normally open CBs. The data of WTG, GTGs, distribution lines and loads are given in [13].

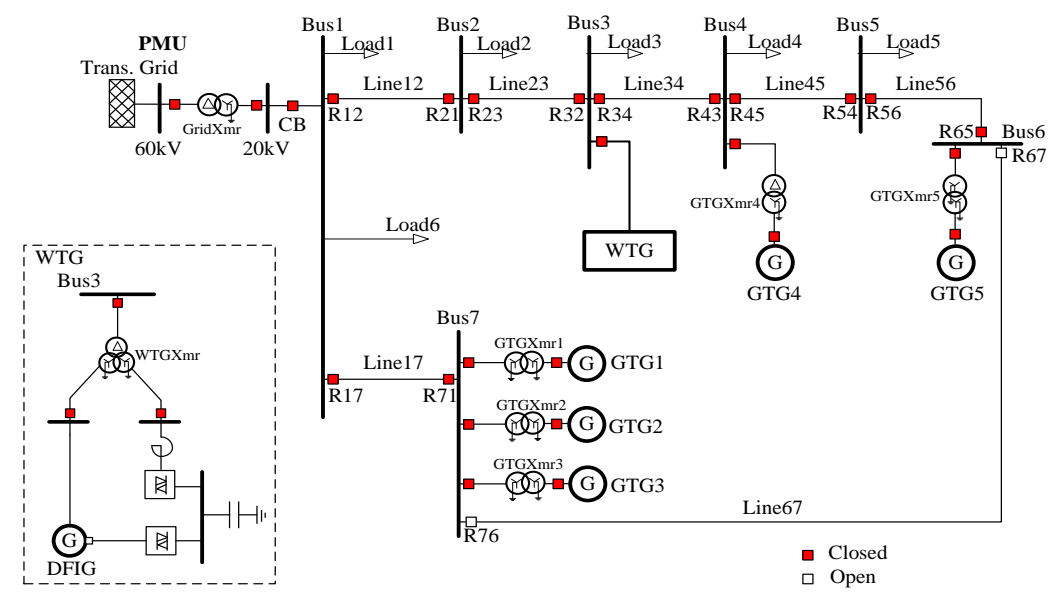

Fig. 1. Test distribution system.

\section{Proposed Methodology}

\subsection{Fault current analysis}

Future distribution systems trend to have ability to be operated in islanded mode for higher reliability. Compared with the grid-connected mode, in which the transmission system provides significant part of the short-circuit power, the short-circuit current in islanded mode is significantly less. The maximum 3phase bolted fault current at the end of each protection zone for grid connected mode and islanded mode are shown in Fig. 2. The difference of short-circuit current only has impacts on the protection settings of forward relays in distribution feeders, (i.e. R12, R23, R34, R45, R56 and R17).

The short circuit current reduces when some of the DG units are disconnected. Fig. 3 compares the maximum short circuit current of 3-phase faults at the beginning of each protection zone when some of DG units are disconnected. The difference of short-circuit current only have impacts on the protection settings of the backward relays in the distribution feeders, (i.e. R17, R21, R32, R43, R54 and R65).

Furthermore, in order to support important load (i.e. Load 5) in the end of distribution feeder, the 
network can be meshed by closing the normally open CBs of Line 67. In these cases, all power resources can contribute the short circuit power from two directions. Therefore, meshing of the network changes the short circuit current seen by most of the forward and backward relays, irrespective of grid-connected mode or islanded mode, as shown in Fig. 4 and Fig. 5, respectively.

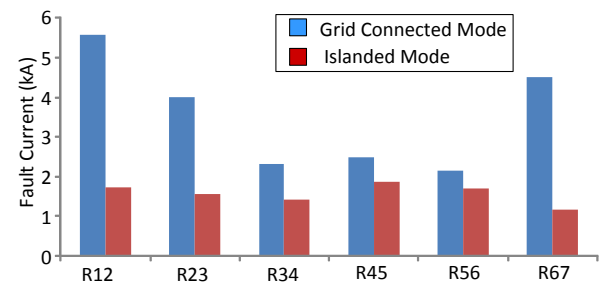

Fig. 2. Max. 3-ph fault current of radial topology.

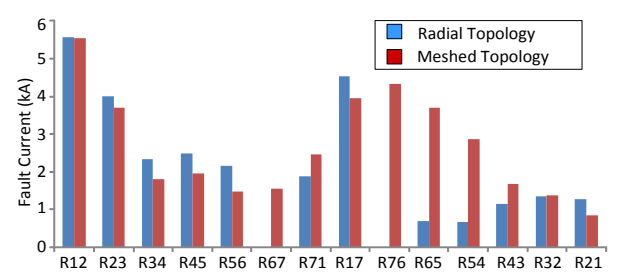

Fig. 4. Max. 3-ph fault current in grid connected mode.

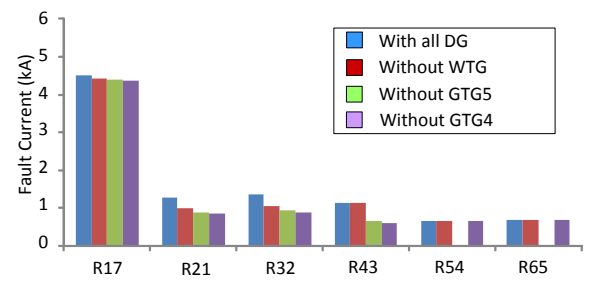

Fig. 3. Max. 3-ph fault current with/without DG integrated.

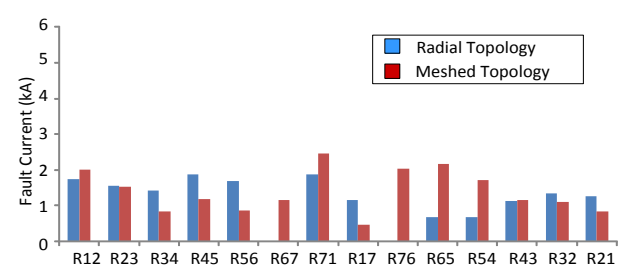

Fig. 5. Max. 3-ph fault current in islanded mode.

\subsection{Inverse-time directional over current relay (DOCR) protection}

Inverse-time directional overccurent relay (DOCR) protection is adopted in the distribution system, which consists of two parts, instantaneous part and inverse-time part. For higher fault current $(I>>)$, the relay pick-up time is fixed to $t_{i n s}$. While for lower fault current (I>), the operation time is inversely changed with the fault current. Compared with traditional definite time DOCR, inverse-time DOCR has much shorter tripping time to reduce the risk of large scale DG disconnections. In this study, the operating time-current curve of inverse-time DOCR follows normal inverse curve in IEC 60255-3 [15], given by

$$
t=\frac{0.14 T_{p}}{\left(I / I_{p}\right)^{0.02}-1}(\mathrm{sec})
$$

where $I, I_{p}$ and $T_{p}$ are fault current, pick-up current, and time dial settings. The instantaneous pick-up current $I_{\text {ins }}$ is set as 1.05 times of the max short circuit current at the end of protection zones. Therefore, $I_{p}$, $T_{p}, I_{i n c}$ are the three configurable setting values for an adaptive DOCR to fit for current operating mode.

\subsection{Architecture of multi-agent system}

Multi-agent system (MAS), also known as "self-organization system" is defined as a computerized system composed of many intelligent agents cooperatively adapting to the environment. Each autonomous agent can freely participate in or leave a group to cooperate with other agents with the help of communication [16]. The proposed MAS based adaptive protection scheme adopts three-level hierarchical architecture, which is built up with centralized control level, cooperation society level and distributed agent level, as shown in Fig. 6. These agents are geographically distributed in a number of intelligent electronic devices (IEDs), which have basic functions, such as computation, I/O interface, communications features, and decision making capability.

MAS in the distribution network consist of Relay Agents Society (RAS), DG Agents Society (DGAS) as well as Equipment Agents Society (EAS). Agents in the MAS can communicate with each other not only within the same agent society, but also between different agent societies.

Each relay is regarded as one relay agent (RA), which searches for relevant information from the communication bus and interacts with other agents. With the help of MAS, RAs not only fulfill the 
primary protection and back up protection with better performance than conventional methods, but also detect events, such as CB failures, CB mal-operations and DG connection status, etc..

Every DG unit is considered as a DG agent (DGA). In MAS environment, DG units not only communicate with the local RA to provide the message of connection status, but also transmit important information, such as status and power generation, to DGAS.

The equipment agent (EA) includes CB agents, current transformer (CT) agents, voltage transformer (VT) agents, etc. These agents can send local important information, such as mal-function, mis-operation, alarm signals etc. to local RAs, and also can communicate with other EAs in the EAS to provide back up and coordination function.

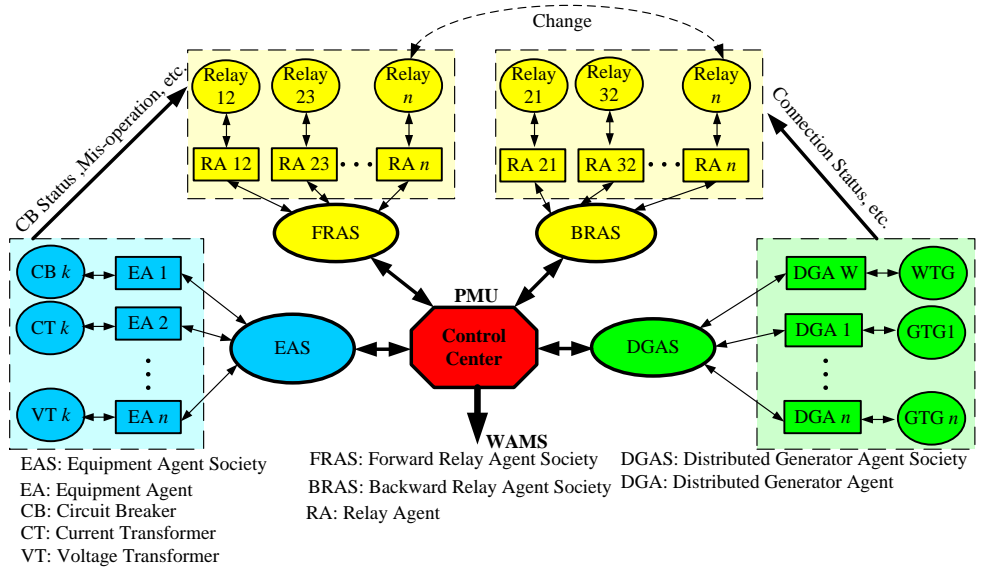

Fig. 6. Architecture of MAS based adaptive protection system.

\subsection{Mobile Multi-Agent System}
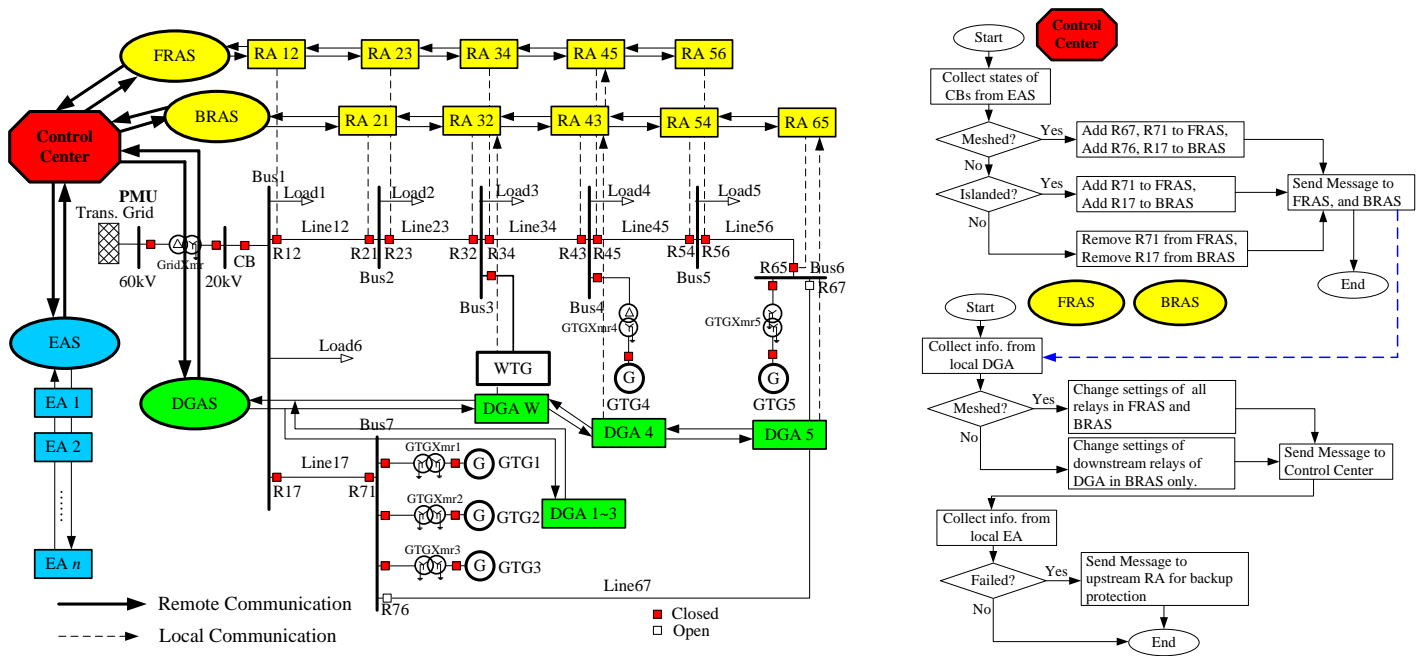

Fig. 7. Structure of MAS in the test distribution system.

Fig. 8. Block diagram of mobile MAS.

Fig. 7 shows MAS in the test distribution system. Based on geographical distribution of agents in the distribution system, the communication can be divided into local communication and remote communication. Mobile MAS is adopted in this approach. As shown in Fig. 8, intelligent electric devices e.g. PMU, located in the substation of transmission system, can serve as the control center of the distribution system. The control center collects the states of CBs from EAS to detect the topology of the network, then to correctly change the classification of relays in Forward Relay Agent Society (FRAS) and Backward Relay Agent Society (BRAS). The protection settings of relays are calculated and stored offline in RAs. Then RAs receive the requests to adopt correct settings to adapt to current operating states. 
The protection settings must coordinate with each other in the same society, as shown in Table 1, in which " $\Rightarrow$ " means the back-up relationship between protection relays, e.g. "R12 $\Rightarrow$ R23" means R12 is the back up protection for R23. The adoption of mobile MAS aims at reducing the communication delay for transmitting the request of setting adjustment.

Table 1. Coordination of FRAS and BRAS with respect to different topologies

\begin{tabular}{lcc}
\hline \hline \multicolumn{1}{c}{ Topology } & Coordination of FRAS & Coordination of BRAS \\
\hline Radial Grid-connected & $R 12 \Rightarrow R 23 \Rightarrow R 34 \Rightarrow R 45 \Rightarrow R 56$ & $R 65 \Rightarrow R 54 \Rightarrow R 43 \Rightarrow R 32 \Rightarrow R 21$ \\
Radial Islanded & $R 71 \Rightarrow R 12 \Rightarrow R 23 \Rightarrow R 34 \Rightarrow R 45 \Rightarrow R 56$ & $R 65 \Rightarrow R 54 \Rightarrow R 43 \Rightarrow R 32 \Rightarrow R 21 \Rightarrow R 17$ \\
Meshed Grid-connected & $R 12 \Rightarrow R 23 \Rightarrow R 34 \Rightarrow R 45 \Rightarrow R 56 \Rightarrow R 67 \Rightarrow R 71$ & $R 17 \Rightarrow R 76 \Rightarrow R 65 \Rightarrow R 54 \Rightarrow R 43 \Rightarrow R 32 \Rightarrow R 21$ \\
Meshed Islanded & $R 12 \Rightarrow R 23 \Rightarrow R 34 \Rightarrow R 45 \Rightarrow R 56 \Rightarrow R 67 \Rightarrow R 71$ & $R 17 \Rightarrow R 76 \Rightarrow R 65 \Rightarrow R 54 \Rightarrow R 43 \Rightarrow R 32 \Rightarrow R 21$ \\
\hline \hline
\end{tabular}

\section{Case Study}

According to Danish grid code, WTG should withstand faults for at least 100ms without disconnection [17]. In this case study, it is assumed that the instantaneous pick-up time $T_{\text {ins }}$ is $10 \mathrm{~ms}$ to detect the fault and relays take another $10 \mathrm{~ms}$ to close its trip contacts. CBs take $60 \mathrm{~ms}$ to clear the fault. So the minimum tripping time is $80 \mathrm{~ms}$ after short-circuit. The communication is simulated by DIgSILENT Simulation Language (DSL) in the DIgSILENT. An Ethernet LAN communication network with ring topology is considered in the distribution system, so that communication delay between any adjacent agents can be assumed to be the typical value of $3.707 \mathrm{~ms}$ [8]. The connection status of DG units will definitely decide the protection settings of its downstream relays and the request signal is transmitted along the feeders in the distribution network. Table 2 shows communication delay of transmitting the new settings throughout the network after the change of DG connection status.

The change of connection status of GTG5 is demonstrated in this study. The instantaneous pick-up current $I_{i n s}$, reverse-time pick-up current $I_{p}$, time dial setting $T_{p}$ of all relays with GTG5 connected and disconnected are listed in Table 3. Accordingly, the time-overcurrent plot of forward relays and backward relays are shown in Fig. 9 and Fig. 10. The time over-current curves of the BRAS in the Fig. 9 (b) and Fig. 10 (b) cross each other but they do not lack of selectivity, owing to the in-feed current from WTG, GTG4 and GTG5 along the distribution feeder.

Table 2. Communication delay for transmitting new protection settings throughout the network.

\begin{tabular}{lcccc}
\hline \hline DG connection states (network topology) & $\begin{array}{c}\text { Msg. of protection settings } \\
\text { communicate in FRAS of MAS }\end{array}$ & $\begin{array}{c}\text { Delay } \\
\text { (ms) }\end{array}$ & $\begin{array}{c}\text { Msg. of Protection Settings } \\
\text { communicate in BRAS of MAS }\end{array}$ & $\begin{array}{c}\text { Delay } \\
\text { (ms) }\end{array}$ \\
\hline WTG disconnected (meshed) & $\mathrm{R} 34, \mathrm{R} 45, \mathrm{R} 56, \mathrm{R} 67, \mathrm{R} 71, \mathrm{R} 12, \mathrm{R} 23$ & 11.121 & $\mathrm{R} 32, \mathrm{R} 21, \mathrm{R} 17, \mathrm{R} 76, \mathrm{R} 65, \mathrm{R} 54, \mathrm{R} 43$ & 11.121 \\
GTG4 disconnected (meshed) & $\mathrm{R} 45, \mathrm{R} 56, \mathrm{R} 67, \mathrm{R} 71, \mathrm{R} 12, \mathrm{R} 23, \mathrm{R} 34$ & 11.121 & $\mathrm{R} 43, \mathrm{R} 32, \mathrm{R} 21, \mathrm{R} 17, \mathrm{R} 76, \mathrm{R} 65, \mathrm{R} 54$ & 11.121 \\
GTG5 disconnected (meshed) & $\mathrm{R} 56, \mathrm{R} 67, \mathrm{R} 71, \mathrm{R} 12, \mathrm{R} 23, \mathrm{R} 34, \mathrm{R} 45$ & 11.121 & $\mathrm{R} 32, \mathrm{R} 21, \mathrm{R} 17, \mathrm{R} 76, \mathrm{R} 65, \mathrm{R} 54, \mathrm{R} 43$ & 11.121 \\
WTG disconnected (radial, islanded) & $\mathrm{R} 34, \mathrm{R} 45, \mathrm{R} 56$ & 7.414 & $\mathrm{R} 32, \mathrm{R} 21, \mathrm{R} 17$ & 7.414 \\
GTG4 disconnected (radial, islanded) & $\mathrm{R} 45, \mathrm{R} 56$ & 3.707 & $\mathrm{R} 43, \mathrm{R} 32, \mathrm{R} 21, \mathrm{R} 17$ & 11.121 \\
GTG5 disconnected (radial, islanded) & $\mathrm{n} / \mathrm{a}$ & $\mathrm{n} / \mathrm{a}$ & $\mathrm{R} 65, \mathrm{R} 54, \mathrm{R} 43, \mathrm{R} 32, \mathrm{R} 21, \mathrm{R} 17$ & 11.121 \\
WTG disconnected (radial, grid-connected) & $\mathrm{R} 34, \mathrm{R} 45, \mathrm{R} 56$ & 7.414 & $\mathrm{R} 32, \mathrm{R} 21$ & 3.707 \\
GTG4 disconnected (radial, grid-connected) & $\mathrm{R} 45, \mathrm{R} 56$ & 3.707 & $\mathrm{R} 43, \mathrm{R} 32, \mathrm{R} 21$ & 7.414 \\
GTG5 disconnected (radial, grid-connected) & $\mathrm{n} / \mathrm{a}$ & $\mathrm{n} / \mathrm{a}$ & $\mathrm{R} 65, \mathrm{R} 54, \mathrm{R} 43, \mathrm{R} 32, \mathrm{R} 21$ & 11.121 \\
\hline \hline
\end{tabular}

Table 3. Time overcurrent settings with and without GTG5.

\begin{tabular}{|c|c|c|c|c|c|c|c|c|c|c|c|c|c|c|c|}
\hline \multicolumn{8}{|c|}{ Protection Settings of Grid-connected \& Radial w GTG5 } & \multicolumn{8}{|c|}{ Protection Settings of Grid-connected \& Radial w/o GTG5 } \\
\hline Relay & $I_{p}(\mathrm{~A})$ & $T_{p}(\mathrm{sec})$ & $I_{\text {ins }}(\mathbf{A})$ & \begin{tabular}{|l|l|} 
Relay \\
\end{tabular} & $I_{p}(\mathrm{~A})$ & $T_{p}(\mathrm{sec})$ & $\boldsymbol{I}_{\text {ins }}(\mathbf{A})$ & Relay & $I_{p}(\mathbf{A})$ & $T_{p}(\mathrm{sec})$ & $\boldsymbol{I}_{\text {ins }}(\mathbf{A})$ & Relay & $I_{p}(\mathbf{A})$ & $T_{p}(\mathrm{sec})$ & $I_{\text {ins }}(\mathbf{A})$ \\
\hline $\mathbf{R 1 2}$ & 440 & 0.1669 & 5532 & $\mathbf{R 2 1}$ & 350 & 0.0243 & 1075 & $\mathbf{R} 12$ & 440 & 0.1669 & 5532 & $\mathbf{R 2 1}$ & 210 & 0.0356 & 945 \\
\hline $\mathbf{R} 23$ & 350 & 0.1275 & 4210 & R32 & 350 & 0.0427 & 1140 & R23 & 350 & 0.1275 & 4210 & R32 & 210 & 0.0615 & 987 \\
\hline R34 & 260 & 0.0848 & 2646 & R43 & 260 & 0.0629 & 900 & R34 & 260 & 0.0848 & 2646 & R43 & 130 & 0.0986 & 682 \\
\hline R45 & 130 & 0.0649 & 2465 & R54 & 130 & 0.0862 & 488 & R45 & 130 & 0.0649 & 2465 & R54 & - & - & - \\
\hline R56 & 130 & 0.0208 & 2208 & R65 & 130 & 0.1073 & 500 & R56 & 130 & 0.0208 & 2208 & R65 & - & - & - \\
\hline R71 & 390 & 0.1232 & 1426 & R17 & 390 & 0.0183 & 4746 & R71 & 390 & 0.1232 & 1426 & R17 & 390 & 0.0183 & 4606 \\
\hline
\end{tabular}


The operating state is the grid-connected mode with radial topology. The extreme scenario is two 3-ph faults with resistance of $0.05 \Omega$ close to the end $(90 \%)$ of Line43 and Line56, taking place at the same time $t=1000 \mathrm{~ms}$. The protection settings are restored for adaptive adjustment for DG disconnection, as listed in Table 3. Fig. 11 shows actual states of CBs for conventional pilot protection, in which WTG is tripped at $t=1262 \mathrm{~ms}$. However, with the help of MAS-based adaptive protection, WTG still remains connected to the network, due to quick change of protection settings to adapt to the new operating states with the help of MAS, as shown in Fig. 12. The sequences of events in the case of conventional pilot protection and MAS-based adaptive protection are compared in Table 4 and the signals transmitted in both of communication-based protections are shown in Fig.13 and Fig. 14.
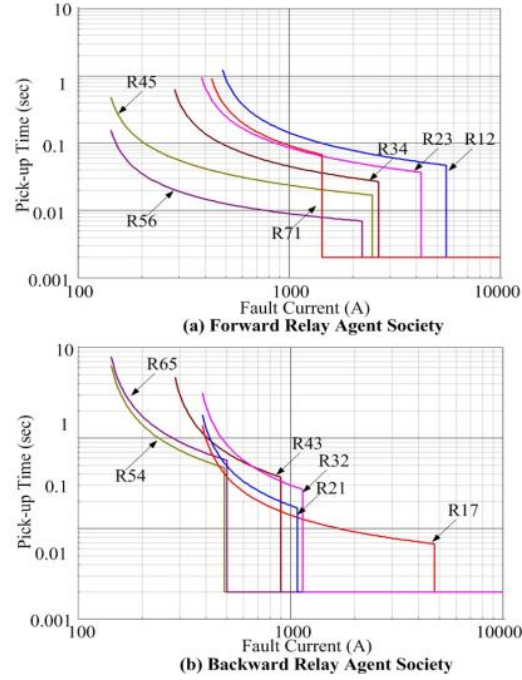

Fig. 9. Time-overcurrent plot with GTG5 connected.

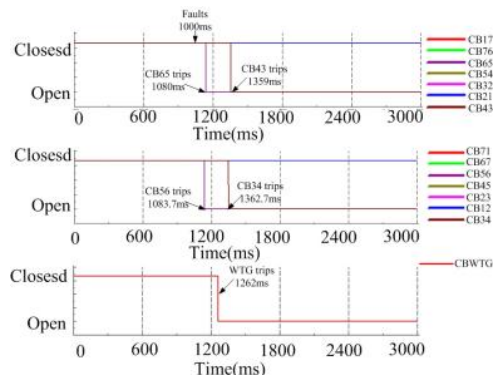

Fig. 11. States of CBs in conventional pilot protection.

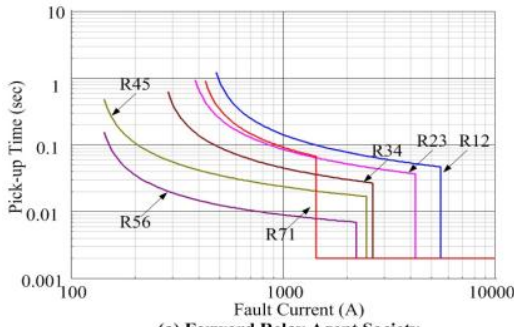

(a) Forward Relay Agent Society

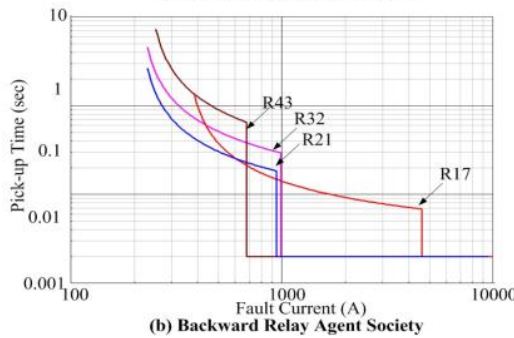

Fig. 10. Time-overcurrent plot with GTG5 disconnected.
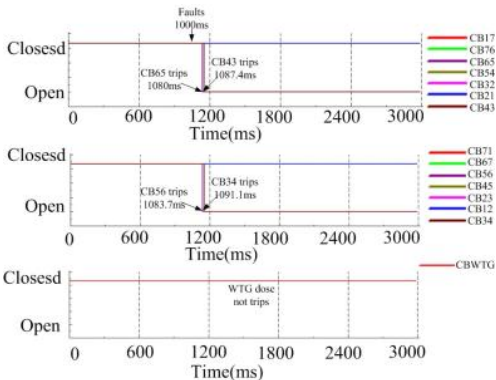

Fig. 12. States of CBs in MAS-based adaptive protection.

Table 4. Sequence of events of the conventional pilot protection and MAS-based adaptive protection

\begin{tabular}{lc||lc}
\hline \hline \multicolumn{1}{c|}{ Conventional pilot protection } & Time $(\mathbf{m s})$ & \multicolumn{1}{c}{ MAS-based adaptive protection } & Time (ms) \\
\hline 3-ph short circuit fault at Line34 and Line56 & 1000 & 3-ph short circuit fault at Line34 and Line56 & 1000 \\
R65 is picked up by instantaneous DOCR & 1010 & R65 is picked up by instantaneous DOCR & 1010 \\
R65 sends tripping signal to R56 & 1010 & R65 sends tripping signal to R56, and requests & 1010 \\
& & for adjusting protection setting in BRAS & 1013.7 \\
R56 receives the tripping signal from R65 & 1013.7 & R56 receives the tripping signal from R65 & 1013.7 \\
CB65 trips, GTG5 trips & 1080 & R54 receives the request for adjust settings & 1017.4 \\
CB56 trips & 1083.7 & R43 receives the request for adjust settings & 1027.4 \\
WTG trips & $\mathbf{1 2 6 2}$ & R43 is picked up by instantaneous DOCR & 1027.4 \\
R34 is picked up by reverse-time DOCR & 1289 & R43 sends tripping signal to R34 & 1031.1 \\
R34 sends tripping signal to R43 & 1289 & R34 receives the tripping signal from R43 & 1080 \\
R43 receives the tripping signal from R34 & 1292.7 & CB65 trips, GTG5 trips & 1083.7 \\
CB34 trips & 1359 & CB56 trips & 1087.4 \\
CB43 trips & 1362.7 & CB43 trips & CB34 trips \\
& & & 1091.1 \\
\hline \hline
\end{tabular}




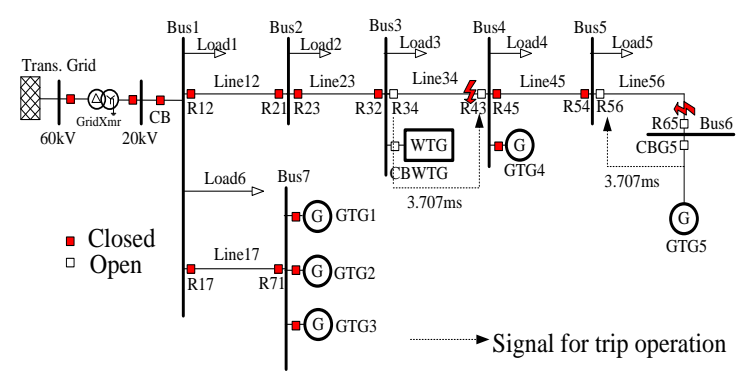

Fig. 13. Signals in conventional pilot protection.

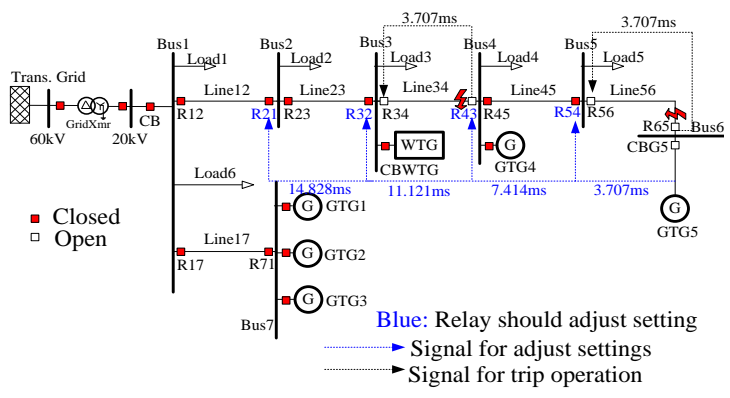

Fig. 14. Signals in MAS-based adaptive protection.

\section{Conclusion}

A coordinative adaptive protection based on MAS is proposed, which adapts itself for multiple variations, i.e. operating states of DG units, network topology, in DG integrated distribution system. The cooperative nature of proposed MAS based protection scheme adjusts settings of protection equipments to fits for current states at high speed. The proposed protection scheme has been applied to a real distributions system and the results have demonstrated the effectiveness of MAS based protection scheme.

\section{Acknowledgements}

The authors would like to acknowledge financial support from the project "Development of a Secure, Economic and Environmentally-friendly Modern Power Systems" (DSF 09-067255).

\section{References}

[1] Anderson PM. Power System Protection. McGraw-Hill, 1999.

[2] Rockefeller GD, Wagner CL, Linder JR, Hicks KL, Rizy DT. Adaptive transmission relaying concepts for improved performance. IEEE Trans. on Power Delivery, 1988, 3(4):1446-1458.

[3] Wan H, Li KK, Wong KP. An adaptive multi-agent approach to protect relay coordination with distributed generators in industrial power distribution system. IEEE Trans. on Ind. Appl., 2010, 46(5):2118-2124.

[4] Buse DP, Wu QH. Mobile agents for remote control of distributed systems. IEEE Trans. on Ind. Electron., 2004, 51(6):11421149.

[5] Baxevanos IS, Labridis DP. Implementing multiagent systems technology for power distribution network control and protection management. IEEE Trans. on Power Delivery, 2007, 22(1):433-443.

[6] Park SJ, Lim JT. Modeling and control of agent-based power protection systems using supervisors. IEE Proc. Control Theory Appl., 2006, 153(1):92-98.

[7] Shi S, Jiang B, Dong X, Bo Z. Protection of microgrid. Presented at: the 10th IET Conf. on Developments in Power System Protection, Manchester, 2010.

[8] Wei M, Chen Z. Distribution system protection with communication technologies. Presented at: IECON, Glendale, 2010.

[9] Bo Z. Adaptive noncommunication protection for power lines BO scheme 1 - the delayed operation approach. IEEE Trans. on Power Delivery, 2002, 17(1):85-91.

[10] Bo Z. Adaptive noncommunication protection for power lines BO scheme 2 - the instant operation approach. IEEE Trans. on Power Delivery, 2002, 17(1):92-96.

[11] Bo Z. Adaptive noncommunication protection for power lines BO scheme 3 - the accelerated approach. IEEE Trans. on Power Delivery, 2002, 17(1):97-104.

[12] Bo Z, Dong X, Caunce B, Millar R. Adaptive noncommunication protection of double-circuit line system. IEEE Trans. on Power Delivery, 2003, 18(1):43-49.

[13] Mahat P, Chen Z, Jensen BB, Bak CL. A simple adaptive overcurrent protection of distribution system with distributed generation. IEEE Trans. on Smart Grid, 2011, 2(3):428-437.

[14] Liu C, Chen Z, Liu Z. A communication-less overcurrent protection for distribution system with distributed generation integrated. Presented at: Power Electronics for Distributed Generation System, Aalborg, 2012.

[15] IEC 60255-3, Electrical Relays - Single Input Energizing Quantity Measuring Relays with Dependent or Independent Time.

[16] Wooldridge M. An Introduction of Multi Agent Systems, John Wiley \& Sons, 2002.

[17] IEEE Standard for Interconnceting Distributed Resources into Electric Power Systems, IEEE Standard $1547^{\mathrm{TM}}$, Jun, 2013. 